\title{
UTS APLIKASI TEKNOLOGI DIGITAL
}

\author{
NPM $\quad: 16011014$ \\ NAMA : Winny Susanto \\ PRODI : Manajemen \\ FAKULTAS : Ekonomi \\ EMAIL : winny.16011014@student.ubl.ac.id
}

SOAL TUGAS : [1][2][3][4]

1. Buat Program website E-Commerce (Topik Apa saja)

Buatlah 4 page (website dengan nama sebagai berikut)

Page 1 : index.html

Page 2 : pagedetail1.html (menjelaskan tentang detil produk)

Page 3 : pagedetail2.html (menjelaskan tentang detil produk)

Page 4 : profilanda.html

Pada page index.html terdapat Menu untuk link ke page (halaman lain) dan dari page lain dapat ke halaman utama (index.html)

Setiap halaman memiliki tag yang sudah dipelajari

Kirim File tersebut :

Email : robby.yuliendra@ubl.ac.id

Subjek : NPM NAMA UTS SENIN

File Name : NPM NAMA UTS SENIN 


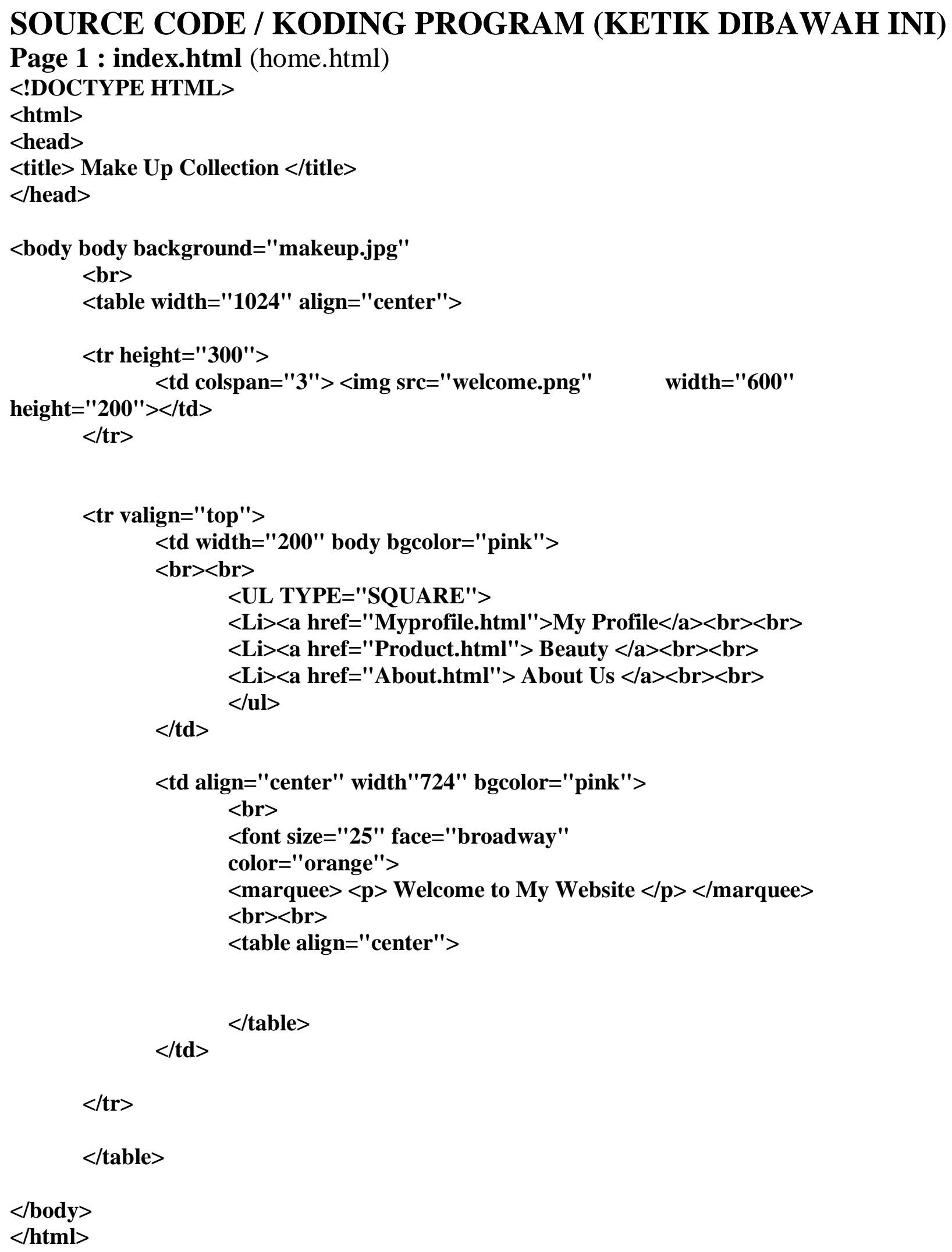




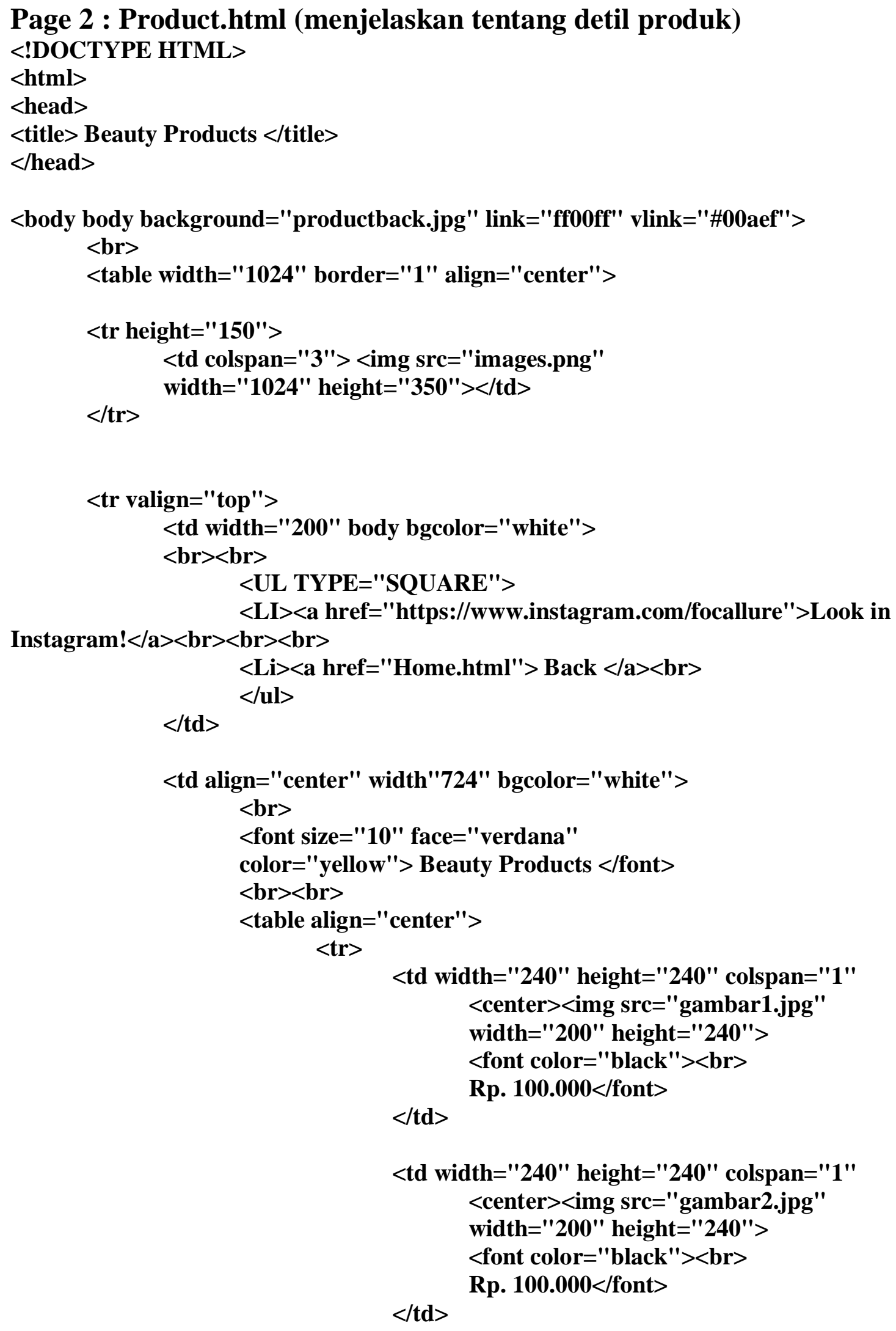




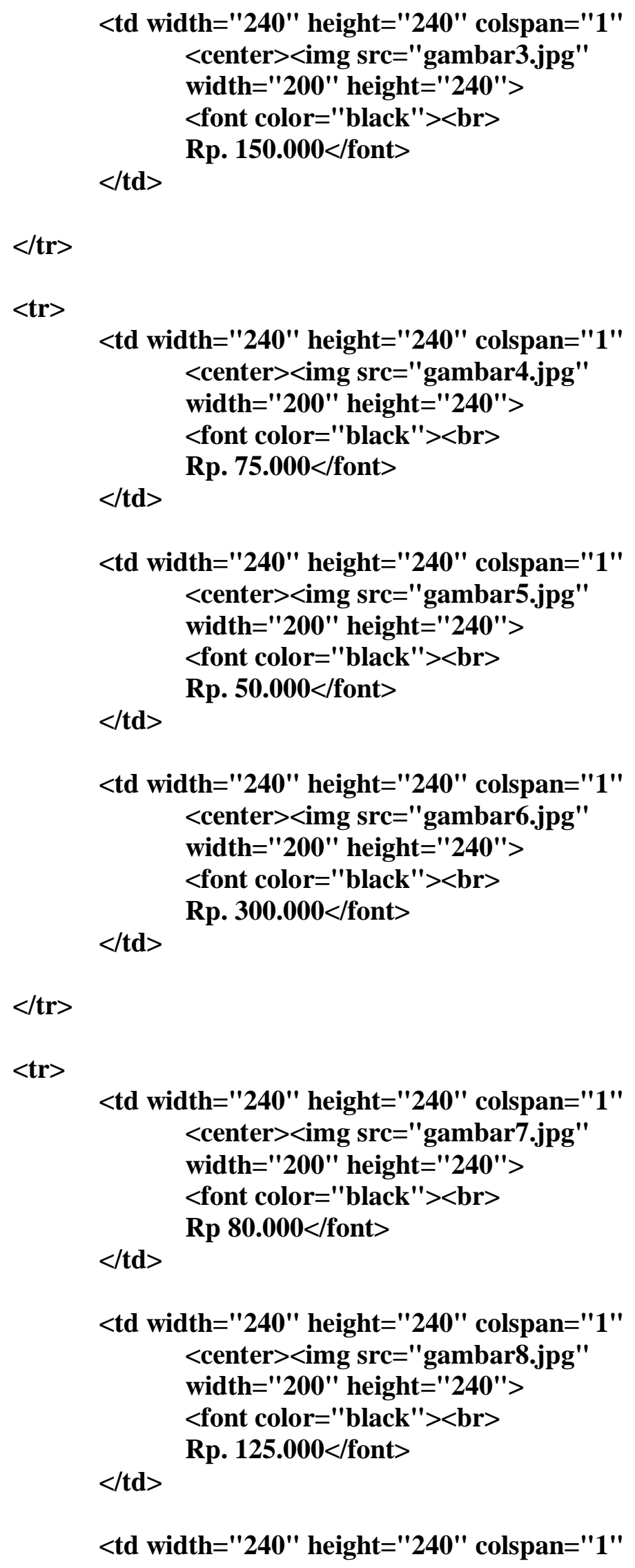




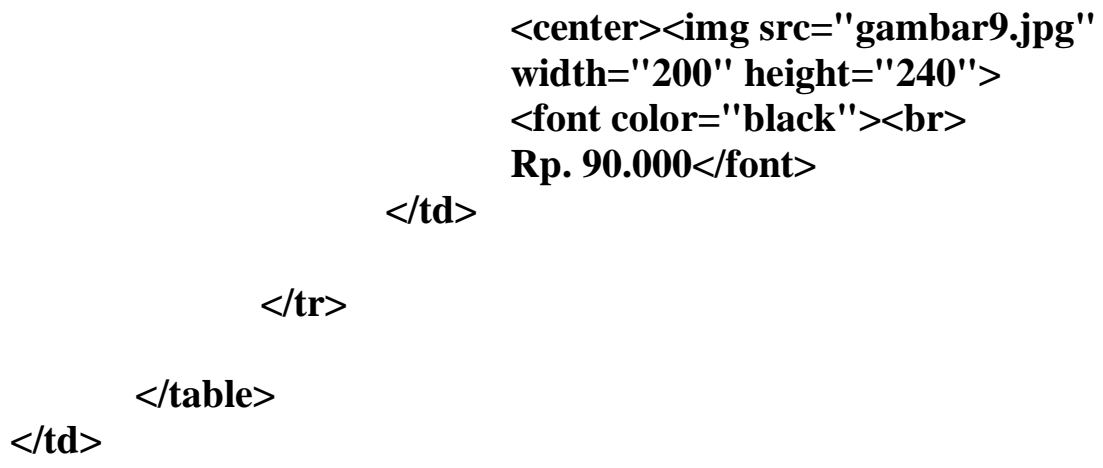




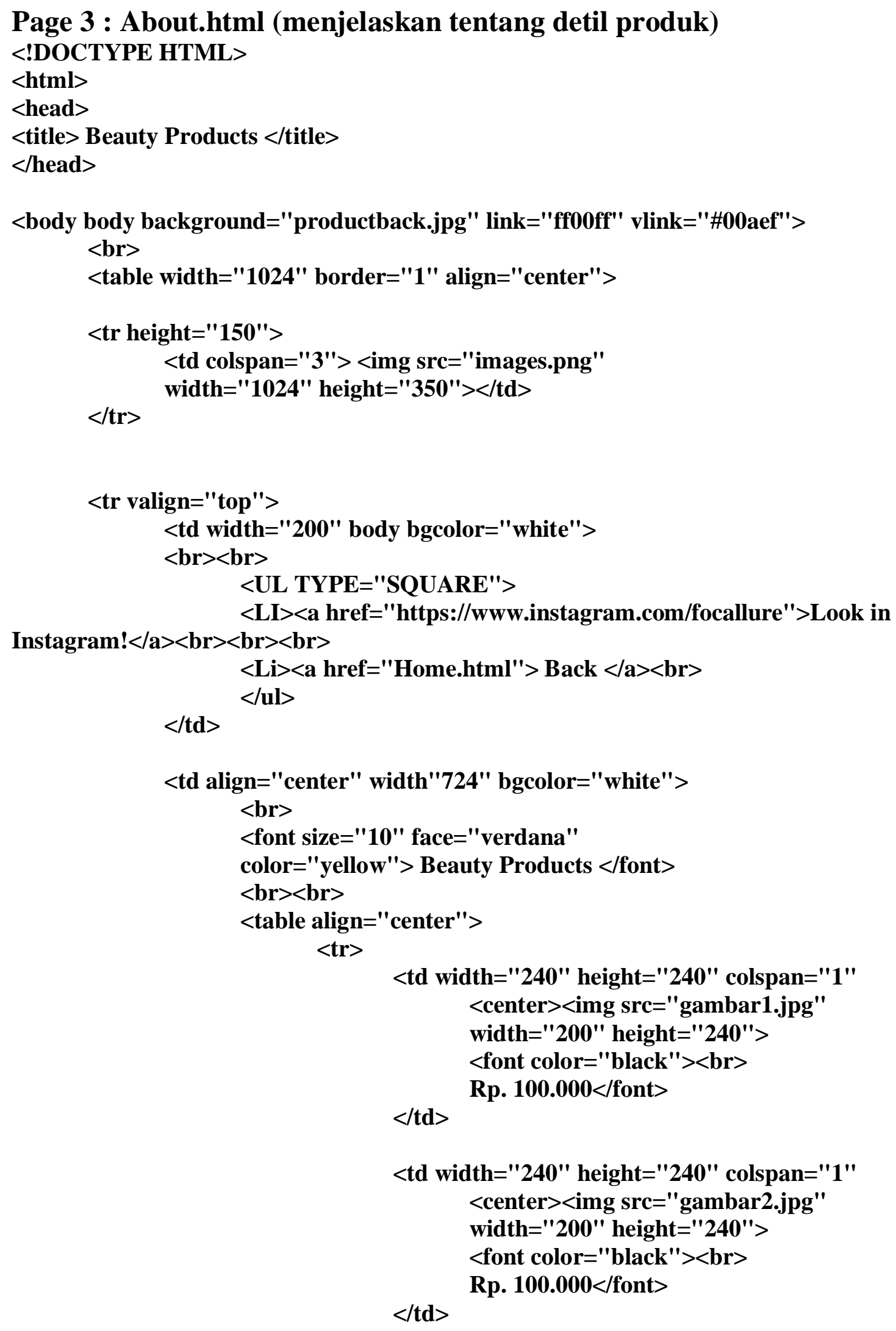




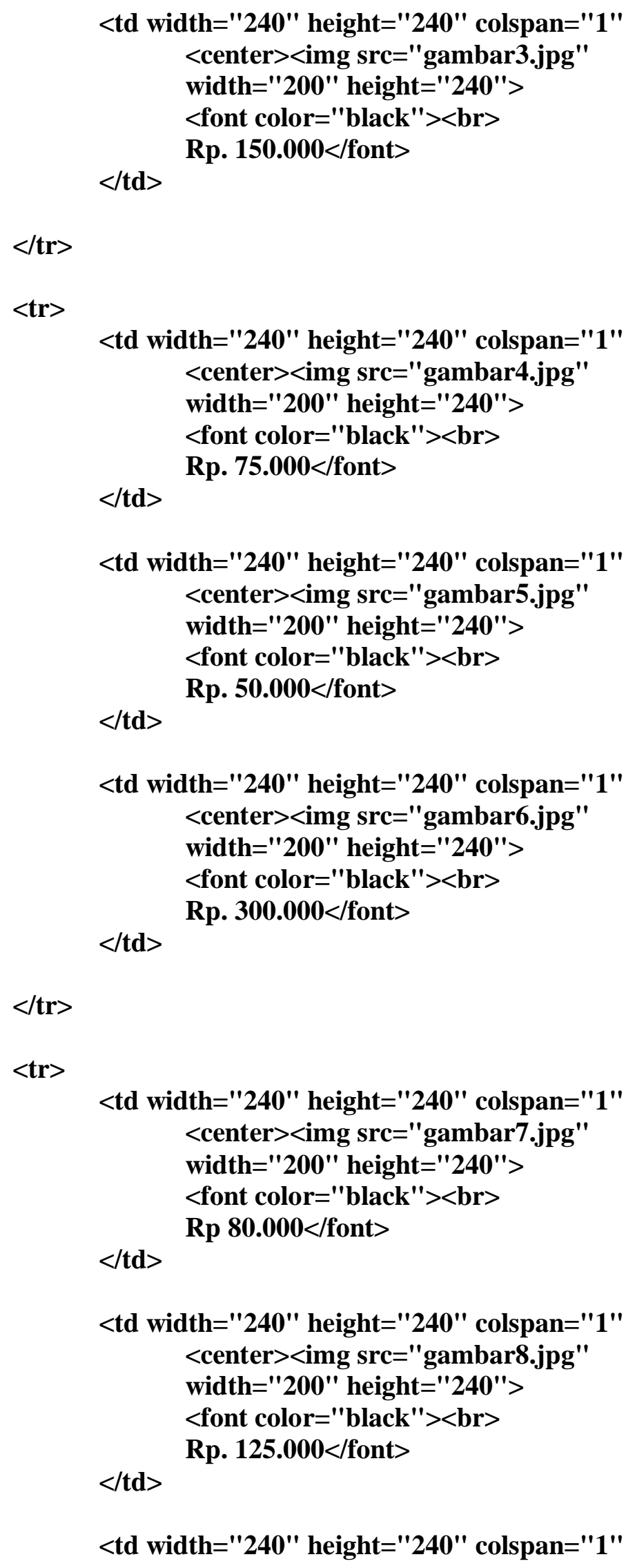




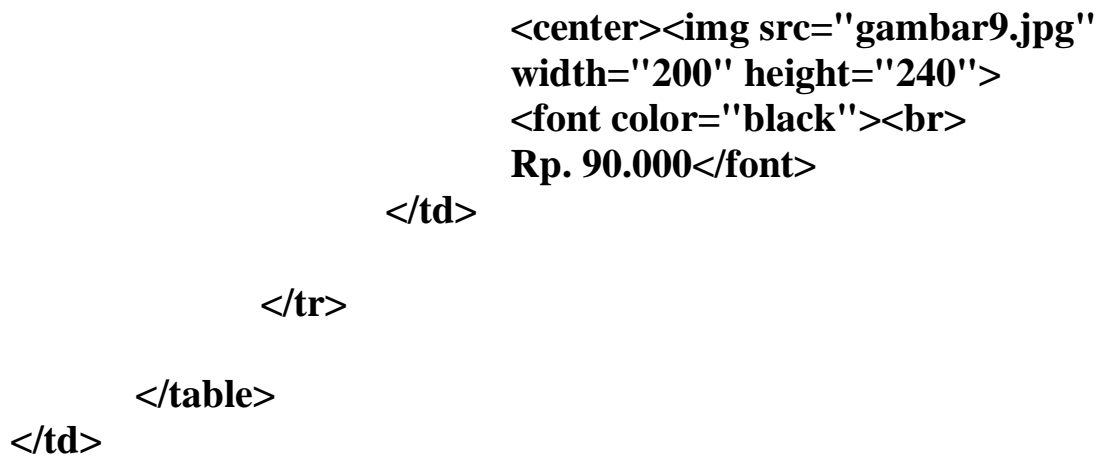




\section{Page 4 : Myprofile.html}

$<$ html>

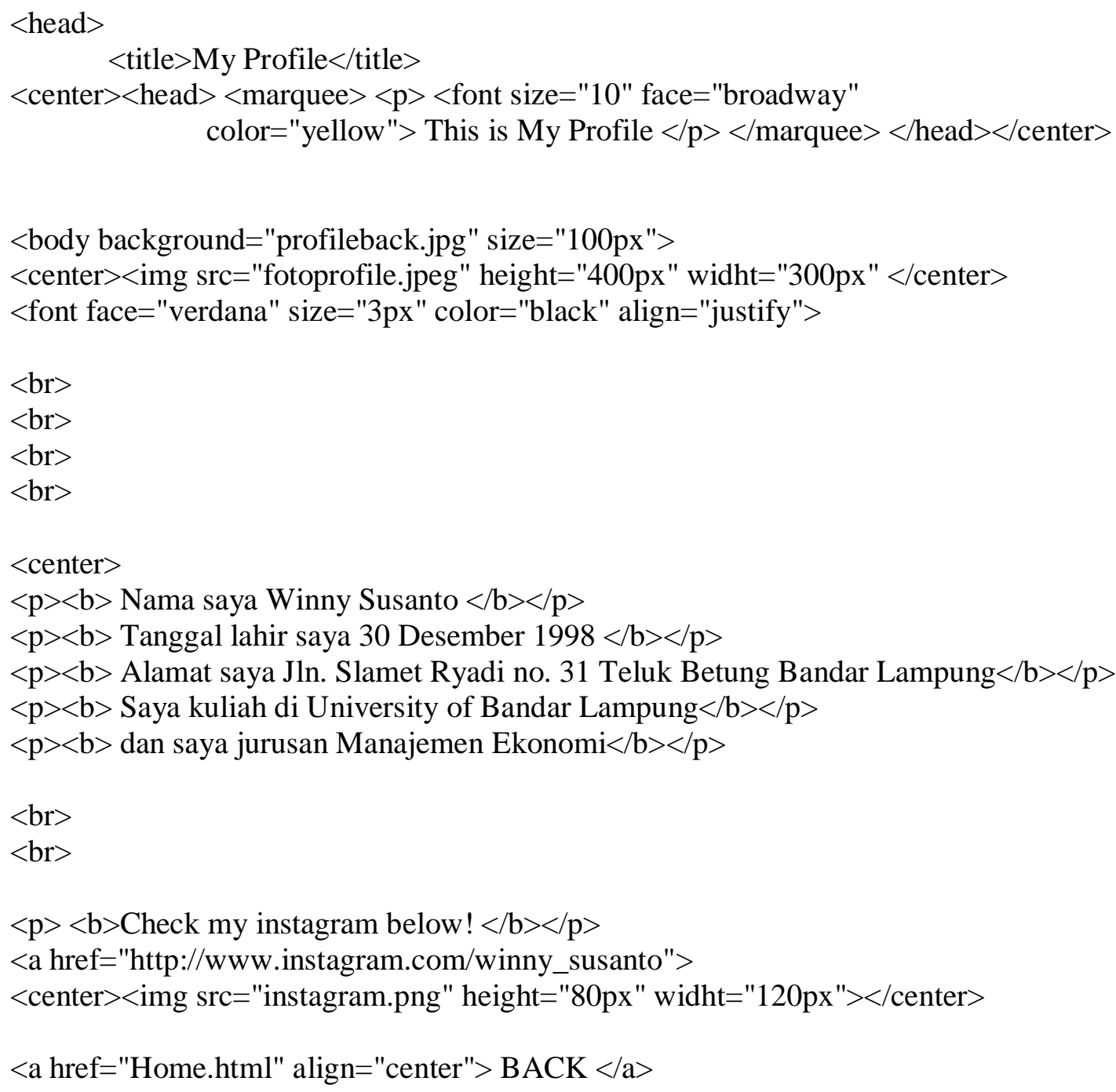




\section{PENJELASAN SOURCE CODE (KETIK DIBAWAH INI)}

<title> Make Up Collection </title>

Untuk memberi judul pada web e-commerce

<body body background="makeup.jpg"

Untuk memberi gambar background pada web e-commerce

<table width="1024" align="center">

Untuk memberi table di web e-commerce

$<\operatorname{tr}$ height="300">

$<$ td colspan="3"><img src="welcome.png" width="600" height="200" ></td $>$ $</$ tr $>$

Untuk memberi semacam gambar banner di dalam web e-commerce

$<$ tr valign="top">

$<$ td width="200" body bgcolor="pink" >

$\langle\mathrm{br}>\langle\mathrm{br}>$

$<$ UL TYPE="SQUARE">

$\langle\mathrm{Li}\rangle\langle$ a href="Myprofile.html" $>$ My Profile $</ \mathrm{a}\rangle\langle\mathrm{br}\rangle\langle\mathrm{br}\rangle$

$\langle\mathrm{Li}\rangle\langle$ a href="Product.html" $>$ Beauty $\langle/ \mathrm{a}\rangle\langle\mathrm{br}\rangle\langle\mathrm{br}\rangle$

$\langle\mathrm{Li}\rangle\langle$ a href="About.html" $>$ About Us $</ \mathrm{a}\rangle\langle\mathrm{br}\rangle\langle$ br $\rangle$

$</$ ul $>$

$</ \mathrm{td}\rangle$

Untuk memberi semacam link ke halaman yang ingin kita tuju

$\langle$ p $\rangle\langle$ b $\rangle$ Check my instagram below $!</ b\rangle\langle/$ p $\rangle$

$<$ a href="http://www.instagram.com/winny_susanto" $>$

$<$ center $><$ img src="instagram.png" height="80px" widht="120px"></center $>$

Untuk memberikan link social media kita di dalam web e-commerce

$<$ font size="25" face="broadway"

color="orange" $>$

$\langle$ marquee $\rangle\langle$ p $>$ Welcome to My Website $\langle$ /p $\rangle<$ /marquee $>$

Untuk memnberikan teks yang berjalan di dalam web e-commerce

$<$ font size="25" face="broadway"

color="orange" $>$

$<$ marquee $>\langle$ p $>$ Welcome to My Website $\langle$ pp $\rangle\langle$ /marquee $>$

Untuk mengcustomisasi font tulisan, ukuran tulisan dan warna tulisan di dalam web ecommerce 
<body bgcolor='pink'>

Untuk memberi warna pada background web e-commerce

<video width="1000" height="600" controls>

<source src="Tutorial.mp4" type="video/mp4">

$</$ video $>$

Untuk memasukan file video ke dalam web e-commerce

<img src="gambar1.jpg"

$$
\begin{aligned}
& \text { width="200" height="240"> } \\
& \text { <font color="black"><br > }
\end{aligned}
$$

Untuk memberikan atau memasukan gambar/foto ke dalam web e-commerce

$\langle\mathrm{p}\rangle$

Tag untuk memasukan kalimat di web e-commerce

$<$ br $>$

Tag untuk memberikan paragraph 


\section{REFERENSI :}

[1] R. Y. Endra, D. S. Aprilita, P. S. Informatika, F. I. Komputer, and U. B. Lampung, "E-REPORT BERBASIS WEB MENGGUNAKAN METODE MODEL VIEW CONTROLLER UNTUK MENGETAHUI PENINGKATAN," pp. 15-22, 2018.

[2] A. Cucus, R. Y. Endra, and T. Naralita, "CHATTER BOT UNTUK KONSULTASI

AKADEMIK DI PERGURUAN TINGGI,” J. Explor., vol. 10, no. 1, pp. 20-25, 2019.

[3] R. Y. Endra and D. Hermawan, "ANALISIS DAN UJI KUALITAS PENGGUNA WEBSITE

TOKOPEDIA.COM MENGGUNAKAN METODE WEBQUAL,” J. Explor., vol. 8, no. 2, pp. 167180, 2017.

[4] R. Y. Endra and D. Prasetya, "Analisis Perbandingan Teknik SEO Antara Google Webmaster Dan

Bing Master Menggunakan Gap Analisis," J. Explor., vol. 8, no. 1, pp. 15-27, 2017. 